\title{
A pleiotropic effect of the single clustered hepatic metastamiRs miR-96-5p and miR-182-5p on insulin-like growth factor II, insulin-like growth factor-1 receptor and insulin-like growth factor-binding protein-3 in hepatocellular carcinoma
}

\author{
REEM AMR ASSAL ${ }^{1}$, HEND MOHAMED EL TAYEBI $^{1}$, KARIM ADEL HOSNY $^{2}$, \\ GAMAL ESMAT $^{3}$ and AHMED IHAB ABDELAZIZ ${ }^{1}$

\begin{abstract}
${ }^{1}$ The Molecular Pathology Research Group, Department of Pharmacology and Toxicology, Faculty of Pharmacy and Biotechnology, German University in Cairo, Cairo $11835 ;^{2}$ Department of General Surgery, Faculty of Medicine, Cairo University; ${ }^{3}$ Department of Endemic Medicine and Hepatology, Cairo University, Cairo 11559, Egypt
\end{abstract}

Received May 22, 2014; Accepted February 6, 2015

DOI: $10.3892 / \mathrm{mmr} .2015 .3382$

\begin{abstract}
MicroRNAs (miRs) have a major role in the pathogenesis of hepatocellular carcinoma (HCC). As the insulin-like growth factor (IGF) axis is a highly tumorigenic pathway in HCC, the present study attempted to target it with miRs. Potential targeting of crucial members of the IGF axis by miRNAs at the 3'-untranslated region (3'-UTR) was predicted using bioinformatic tools, such as microrna.org, Diana lab and Targetscan, while 5'-UTR targeting was predicted using bibiserv software. Expression profiling of obtained miRNAs was performed using quantitative polymerase chain reaction (qPCR) in 22 non-metastatic HCC biopsy samples and 10 healthy tissues. To investigate the impact of miRNAs on their potential downstream targets, transfection of miRNAs was performed in HuH-7 cells and the targets' expression was quantified using qPCR. Transcripts of insulin-like growth factor-1 receptor (IGF-1R), insulin-like growth factor binding protein-3 (IGFBP-3) and IGF-II were found to be potentially targeted at the 5'-UTR and 3'-UTR regions by the single clustered hepatic metastamiRs miR-96-5p and miR-182-5p. The two miRNAs showed a similar expression pattern in HCC tissues compared to those in healthy tissues. Forced expression of miR-96-5p and miR-182-5p in the HCC cell line HuH-7 had inducing effects on IGFBP-3 and IGF-II transcripts. Of
\end{abstract}

Correspondence to: Professor Ahmed Ihab Abdelaziz, The Molecular Pathology Research Group, Department of Pharmacology and Toxicology, Faculty of Pharmacy and Biotechnology, German University in Cairo, New Cairo City, Main Entrance Al Tagamoa Al Khames, Cairo 11835, Egypt

E-mail: ahmed.abdel-aziz@guc.edu.eg

Key words: microRNA-96, microRNA-182, insulin-like growth factor-1R, insulin-like growth factor-II, insulin-like growth factor binding protein 3 note, the two miRs had differential effects on IGF-1R, where miR-96-5p induced IGF-1R mRNA expression and miR-182-5p inhibited its expression. The present study revealed the pleiotropic impact of the single clustered hepatic metastamiRs miR-96-5p and miR-182-5p on IGF-1R, and an inducing effect on IGF-II and IGFBP-3 in hepatocellular carcinoma.

\section{Introduction}

MicroRNAs (miRs) are a novel class of small, non-coding, single-stranded RNA molecules ( 22 nucleotides). They mainly function via base pairing with the 3 '-untranslated region (3'-UTR) of their target mRNAs, thus inhibiting their translation or repressing their levels (1-4). The present study attempted to study the regulation of the 5'UTR and 3'-UTR of insulin-like growth factor-1 receptor (IGF-1R), insulin-like growth factor binding protein-3 (IGFBP-3) and IGF-II, which are members of the IGF axis and have a major role in hepatocarcinogenesis, with the metastamiRs miR-96-5p and miR-182-5p. miR-96 and miR-182 were previously shown to have a role in hepatocellular carcinoma (HCC) (5-11). They belong to the same family (miR-96/182/183 family) and are members of a miRNA cluster that resides in the intergenic region between two protein-coding genes in the chromosomal locus (7q31-34) that is frequently amplified in $\operatorname{HCC}(5,6)$. Gradual increase in miR-96 expression levels was linked to disease progression from normal liver tissues to HCC (6-9). miR-96 was identified as the most strongly associated $\mathrm{HCC}$ recurrence-associated $\mathrm{miR}$ in non-tumor tissues and its expression levels was found to be correlated with the invasive and metastatic potential of HCC cells $(7,10)$. Likewise, miR-182 was significantly upregulated in metastatic HCC tissues compared with that in paired normal tissues. This observed upregulation was significantly associated with intrahepatic metastasis, early recurrence and correlated with the HCC tumor grade (11). However, the expression status of miR-96 and miR-182 in non-metastatic $\mathrm{HCC}$ as well as the underlying mechanism of inducing carcinogenesis has not been investigated previously, to the best of 
our knowledge. Therefore, the present study aimed to explore their impact on the highly conserved IGF tumorigenic pathway.

In HCC tissues, the expression of IGF-II ligand protein was shown to be significantly higher than that in normal liver tissues (12). Similarly, the membrane-bound receptor IGF-1R is overexpressed in various HCC cell lines (13). The expression of IGFBP-3, which modulates the bioavailability of IGF-II ligand, was found to be significantly downregulated in HCC tissues (14). This reduced IGFBP-3 expression may increase the availability of biologically active IGF-II potentiating the proliferative effects (15). Therefore, the present study aimed to explore the impact of the single clustered miRNAs on their downstream targets.

To achieve this aim, screening for miR-96 and miR-182 in non-metastatic HCC tissues was performed followed by manipulation of their expression in the $\mathrm{HCC}$ cell line $\mathrm{HuH}-7$ in an attempt to reveal their impact on the crucial members of the IGF axis (IGF-1R, IGFBP-3 and IGF-II).

\section{Materials and methods}

Study patients. The present study included 22 HCC patients who underwent liver transplant surgery at the Kasr El Einy Hospital, Cairo University, Egypt. Ten liver biopsy specimens were obtained from healthy donors during transplantation. Healthy donors were non-diabetic, non-hypertensive and negative for hepatitis B and C viruses. All participants gave their written informed consent.

The study was approved by the ethical review committee of Cairo University (Cairo, Egypt). The institutional ethics committees approving the protocol of the present study comply with the principles set forth in the international reports and guidelines as stated in the Helsinki Declaration and the International Ethical Guidelines For Biomedical Research Involving Human Subjects issued by the Council For International Organizations of Medical Sciences (CIOMS).

All patients were non-metastatic with no extrahepatic manifestations and no vascular invasion. Most of the patients (68.2\%) had more than one focal lesion as indicated in the pathology report and were subjected to clinical assessment as shown in Table I.

Cell culture. HuH-7 cells were maintained in Dulbecco's modified Eagle's medium (DMEM) supplemented with $4.5 \mathrm{~g} / 1$ glucose, $4 \mathrm{mmol} / \mathrm{l} \mathrm{L}$-glutamine, $10 \%$ fetal bovine serum and mycozap at 1:500 dilution (all Lonza, Basel, Switzerland) at $37^{\circ} \mathrm{C}$ in a $5 \% \mathrm{CO}_{2}$ atmosphere.

Transfection of oligonucleotides. For examining the effect of miR-96-5p and miR-182-5p on IGF-1R, IGF-II and IGFBP-3 transcript expression, HuH-7 cells were transfected with mimics and inhibitors of miR-96-5p and miR-182-5p (Qiagen, Hilden, Germany). All transfection experiments were performed in triplicate using HiPerfect Transfection Reagent (Qiagen) according to the manufacturer's instructions and experiments were repeated three times. Cells that were only exposed to transfection reagent were designated as mock cells, cells transfected with miR-96-5p or miR-182-5p mimics were designated as miR-96-5p cells or miR-182-5p cells, respectively, and cells transfected with miR-96-5p or
Table I. Number/size of focal lesions according to Milan criteria.

\begin{tabular}{|c|c|c|}
\hline Patient ID & Focal lesions (n) & Size of focal lesions $(\mathrm{cm})$ \\
\hline 1 & 3 & $1.5,1,1$ \\
\hline 2 & 1 & 2.5 \\
\hline 3 & 3 & $2,2.5,3$ \\
\hline 4 & 3 & $2,2,3.5$ \\
\hline 5 & 1 & $1.5 \times 2$ \\
\hline 6 & 3 & $3 \times 4,1,1$ \\
\hline 7 & 1 & 4 \\
\hline 8 & 3 & $4,1,1$ \\
\hline 9 & 3 & $1,1,1.5$ \\
\hline 10 & 1 & 2.5 \\
\hline 11 & 2 & $1,1.7$ \\
\hline 12 & 3 & $1,1,1$ \\
\hline 13 & 1 & 3 \\
\hline 14 & 3 & $3,1.5,2$ \\
\hline 15 & 3 & $1,1,4$ \\
\hline 16 & 2 & $3,1.5$ \\
\hline 17 & 2 & $1.5,3$ \\
\hline 18 & 3 & $2.5,2.5,1.5$ \\
\hline 19 & 3 & $1.5,1,1$ \\
\hline 20 & 1 & 2 \\
\hline 21 & 1 & 1.5 \\
\hline 22 & 3 & $3,2.5,1$ \\
\hline
\end{tabular}

miR-182-5p inhibitors were designated as anti-miR-96-5p or anti-miR-182-5p cells, respectively.

Total RNA extraction from liver biopsies and HCC cells. mRNAs and miRs were extracted from liver biopsy specimens and HCC cells. Fresh liver samples (HCC and healthy tissues) were collected during surgery and were snap frozen in liquid nitrogen. The specimens were manually pulverized in liquid nitrogen and $100 \mathrm{mg}$ tissue powder was used for large and small RNA extraction using the mirVana miRNA Isolation kit (Ambion Life Technologies, Waltham, MA, USA) according to the manufacturer's instructions. HCC cells were harvested $48 \mathrm{~h}$ after transfection according to the HiPerfect Transfection Reagent protocol; $150 \mathrm{ng}$ oligonucleotides were used for $\mathrm{HuH}-7$-cell transfection in a six-well plate.

$m i R$ and $m R N A$ quantification. The extracted miRs were reverse transcribed into single stranded complementary DNA (cDNA) using a TaqMan_MicroRNA Reverse Transcription kit (Applied Biosystems Life Technologies) using specific primers for Homo sapiens (hsa)-miR-96-5p, hsa-miR-182-5p as well as RNU6B for normalization. IGF-1R, IGF-II and IGFBP-3 mRNAs were reverse transcribed into cDNA using the High-capacity cDNA Reverse Transcription kit (Applied Biosystems) according to the manufacturer's instructions. Relative expression of miR-96-5p, miR-182-5p and RNU6B as well as IGF-1R, IGF-II, IGFBP-3 and $\beta$-2- microglobulin ( $\beta 2 \mathrm{MG}$; for normalization) was quantified using TaqMan 
Table II. Positions of binding sites of hsa-miR-96-5p and hsa-miR-182-5p to UTRs of IGF-1R, IGFBP-3 and IGF-II (binding sites' positions: 5'-UTR as predicted by bibiserv and 3'-UTR as predicted by microrna.org).

\begin{tabular}{lccccc}
\hline & \multicolumn{3}{c}{ Position of 5'-UTR target regions } & & \multicolumn{2}{c}{ Position of 3'-UTR target regions } \\
\cline { 2 - 5 } miR & IGF-1R & IGFBP-3 & IGF-II & IGF-1R & IGFBP-3 \\
\hline miR-96-5p & $17-29$ & $65-88$ & $630-652$ & $564-592$ & IGF-II \\
miR-182-5p & $14-21$ & $65-78$ & $600-627$ & $5799-5827$ & N/A \\
& & & & $564-592$ & $40-62$ \\
\end{tabular}

hsa, Homo sapiens; UTR, untranslated region, IGFBP, insulin-like growth factor binding protein; IGF-1R, insulin-like growth factor-1 receptor; Pos., position; miR, microRNA; N/A, not available.

Real-Time Q-PCR (assay IDs, 000186, 002334, 001093, Hs00609566_m1, Hs04188276_m1, Hs00365742_g1 and 4310886E, respectively; Applied Biosystems) using a StepOne ${ }^{\mathrm{TM}}$ PCR system (Applied Biosystems). Relative expression was calculated using the $2^{-\Delta \Delta C t}$ method. All PCR reactions including controls were run in duplicate.

Bioinformatics. Putative downstream targets for miR-96-5p and miR-182-5p were predicted using bioinformatics algorithms. 3'-UTR target regions were predicted using microrna.org (www.microrna.org), Diana Lab (www.diana. cslab.ece.ntua.gr/) and TargetScan (www.targetscan.org/), while 5'-UTR target regions were predicted using bibiserv software version 2 (Bielefeld University Bioinformatics Server; http://bibiserv.techfak.uni-bielefeld.de/rnahybrid/submission.html).

Statistical analysis. The Mann-Whitney U test (non-parametric and two-tailed) was performed to compare gene expression between two groups. Pearson's method of parametric statistical correlation was used for correlation analysis, with a Pearson $r>0.3$ indicating a significantly positive correlation and a Pearson $\mathrm{r}<-0.3$ indicating a significantly negative correlation. A P-value $<0.05$ was considered to indicate a statistically significant difference between values. P-values are indicated as ${ }^{* * *} \mathrm{P}<0.001,{ }^{* *} \mathrm{P}<0.01,{ }^{*} \mathrm{P}<0.05$ and ns, statistically not significant. All the data were statistically analyzed using GraphPad Prism 5.00 software (GraphPad, La Jolla, CA, USA).

\section{Results}

miR-96-5p and miR-182-5p screening in liver tissues and HCC cell lines. The relative expression of miR-96 and miR-182 was assessed in the HCC and healthy liver tissues groups, as well as in a $\mathrm{HCC}$ cell line (HuH-7), and its expression was normalized to RNU6B. Expression levels of miR-96 and miR-182 in all HCC biopsy specimens $(n=22)$ were similar to those in healthy tissues $(\mathrm{P}=0.5555$ and $\mathrm{P}=0.1864$, respectively). However, expression of miR-96 in HuH-7 cells was significantly higher than that in healthy tissues $(\mathrm{P}=0.0225)$ (Fig. 1A). In a similar manner, the expression of miR-182-5p in HuH-7 cells showed a significantly higher expression compared to that in healthy tissues and HCC tissues $(\mathrm{P}=0.0002)$ (Fig. 1A).

Correlation analysis between miR-96-5p and miR-182-5p mRNA expression in HCC tissues and healthy controls. miR-96-5p expression was quantified in all healthy and HCC tissues and correlated to miR-182-5p expression in the same patients and healthy controls. Using Pearson's statistical method of correlation, miR-96-5p expression was found to be directly correlated with miR-182-5p expression in all HCC tissues studied, as indicated by a Pearson correlation coefficient of $\mathrm{r}=0.5119$ ( $\mathrm{P}=0.0149$, Fig. 1B). Similarly, in healthy controls, the same direct correlation was observed with a Pearson correlation coefficient of $r=0.6708$ ( $\mathrm{P}=0.0337)$ (Fig. 1B).

Bioinformatics analysis. miR-96-5p and miR-182-5p accession numbers and mature sequences were retrieved using miRBase database (http://www.mirbase.org/). The mature sequences of miR-96-5p and miR-182-5p showed an identical seed sequence (ACGGUU). Using different in silico algorithms for predicting binding to the downstream targets at the 3'-UTR and 5'-UTR positions, miR-96 and miR-182 were shown to target IGF-1R, IGFBP-3 and IGF-II transcripts.

According to microrna.org, Diana Lab and TargetScan, it was shown that miR-96 and miR-182 targeted the 3'-UTR of IGF-1R at two binding sites by the same seed sequence. In silico algorithms further predicted targeting of the IGFBP-3 3'-UTR at one binding site by miR-96 and miR-182. However, the two miRNAs were not predicted to target the 3'-UTR of IGF-II (Table II).

However, miR-96 and miR-182 were shown to target the 5'-UTR of IGF-1R, IGFBP-3 and IGF-II according to bibiserv software (Table II).

Transfection efficiency of miR-96-5p and miR-182-5p oligonucleotides. HuH-7 cells transfected with miR-96 and miR-182 mimics showed upregulation of miR-96 and miR-182 by up to 47.18 - and 98.59 -fold, respectively $(\mathrm{P}=0.0294$ and $\mathrm{P}<0.0001$, respectively) compared to mock-transfected cells (Fig. 2).

Impact of miR-96-5p and miR-182-5p on IGF-1R. Transfection of HuH-7 cells with miR-96 mimics resulted in a significant 
A

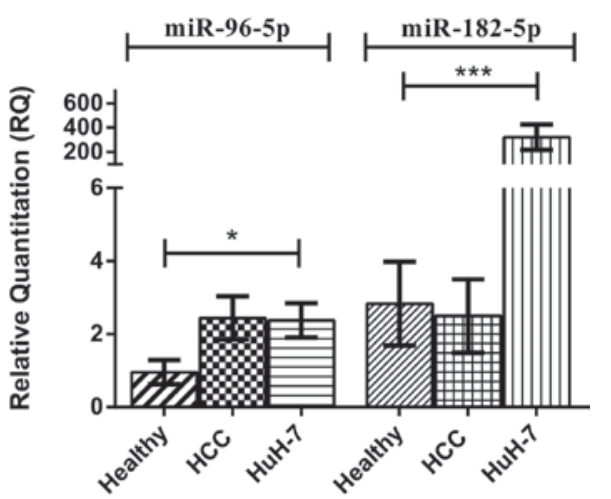

B

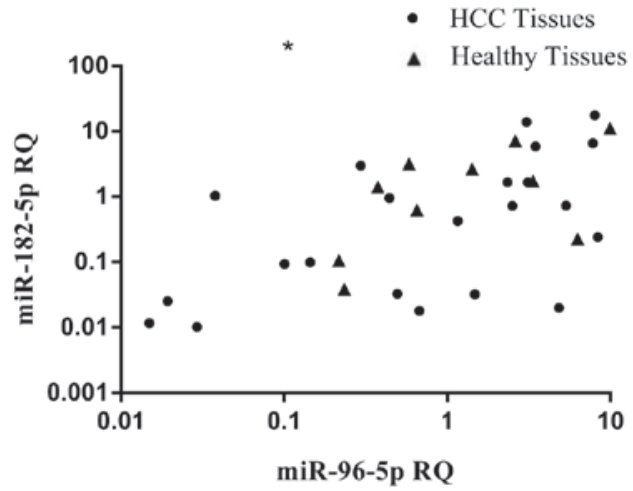

Figure 1. miR-96-5p and miR-182-5p screening in liver tissues and HCC cell lines and their correlation analysis. (A) miR-96-5p expression levels were found to be similar in healthy liver tissues and HCC tissues, while those in the HuH-7 cell line were significantly elevated compared to those in healthy liver tissues. miR-182-5p expression levels were found to be similar in healthy liver tissues and HCC tissues, while being significantly upregulated in the HuH-7 cell line compared to healthy liver tissues and HCC tissues. ${ }^{* * *} \mathrm{P}<0.001,{ }^{*} \mathrm{P}<0.05$. A Mann-Whitney $\mathrm{U}$ test was performed. (B) RQ values of miR-96-5p and miR-182-5p in 22 HCC biopsies and in 10 healthy liver tissues were analyzed using the Pearson method of statistical correlation. A significant direct correlation was revealed between miR-96-5p and miR-182-5p expression in HCC biopsy specimens and in healthy liver tissues. HCC, hepatocellular carcinoma; miR, microRNA; $\mathrm{RQ}$, relative quantitation.

upregulation of IGF-1R mRNA levels $(\mathrm{P}<0.0001)$ compared to those in the mock controls. Of note, inhibitors of miR-96 in HuH-7 caused a significant decrease in IGF-1R mRNA levels compared to those in mimic-transfected cells $(\mathrm{P}<0.0001)$ (Fig. 3A). Of note, transfection of HuH-7 cells with miR-182 mimics showed a significant downregulation of IGF-1R mRNA levels compared to those in mock controls $(\mathrm{P}=0.0267)$. However, inhibitors of miR-182 in HuH-7 did not cause any significant changes in IGF-1R mRNA levels compared to those in mock controls ( $\mathrm{P}=0.1266)$ (Fig. 3A).

Impact of miR-96-5p and miR-182-5p on IGFBP-3. Transfection of HuH-7 cells with miR-96 mimics resulted in a significant upregulation of IGFBP-3 mRNA levels $(\mathrm{P}<0.0001)$ compared to those in mock-transfected controls. While inhibitors of miR-96 caused a significant downregulation of IGFBP-3 mRNA levels in $\mathrm{HuH}-7$ cells compared to those in mock-transfected controls $(\mathrm{P}<0.0001)$ and mimic-transfected cells $(\mathrm{P}<0.0001)$ (Fig. 3B). Transfection of HuH-7 cells with miR-182 caused a significant upregulation of IGFBP-3 mRNA levels compared to those in mock-transfected controls $(\mathrm{P}=0.0357)$. However, inhibitors

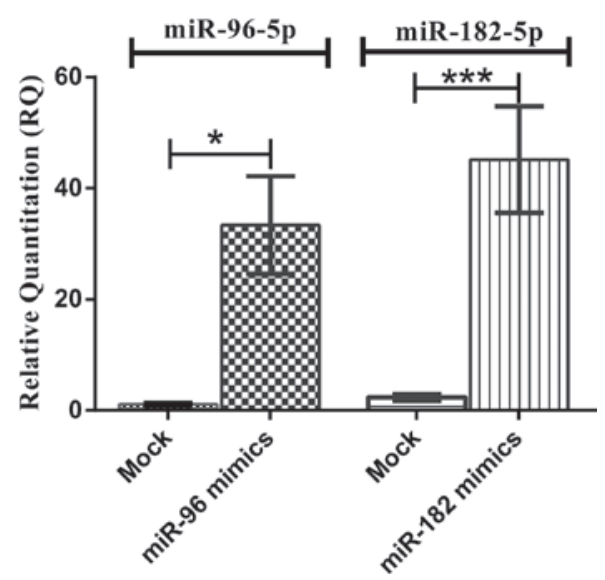

Figure 2. Transfection efficiency of miR-96 and miR-182 oligonucleotides. miR-96 mimics significantly increased miR-96 expression in $\mathrm{HuH}-7$ by up to 47.18 fold compared to transfection with the mock control. miR-182 mimics elevated the expression of miR-182 in HuH-7 cells by up to 98.59 fold compared with mock controls. miR-96 and miR-182 expression was normalized to RNU6B as an internal control. ${ }^{* * *} \mathrm{P}<0.001,{ }^{*} \mathrm{P}<0.05$. The Mann-Whitney U test was performed. miR, microRNA.

of miR-182 did not cause any significant change in IGFBP-3 mRNA levels in HuH-7 cells compared to those in mock cells $(\mathrm{P}=0.7937)$ (Fig. 3B).

Impact of miR-96-5p and miR-182-5p on IGF-II. Transfection of HuH-7 cells with miR-96 mimics resulted in a significant upregulation of IGF-II mRNA levels $(\mathrm{P}<0.0001)$ compared to those in mock-transfected controls. Of note, inhibitors of miR-96 caused a significant downregulation of IGF-II mRNA levels in HuH-7 cells compared to those in the mock-transfected controls $(\mathrm{P}<0.0001)$ and mimic-transfected cells $(\mathrm{P}<0.0001)$ (Fig. 3C). Transfection of $\mathrm{HuH}-7$ cells with miR-182 mimics caused a significant upregulation of IGF-II mRNA levels compared to those in mock-transfected controls $(\mathrm{P}=0.0317)$. Inhibitors of miR-182 did not cause any significant changes in IGF-II mRNA levels in HuH-7 cells compared with those in mock-transfected controls $(\mathrm{P}=0.4762)$ (Fig. 3C).

\section{Discussion}

miR-96-5p and miR-182-5p, which belong to the same family of single clustered miRs, were recently shown to be oncomiRs that promote oncogenesis with an essential role in $\mathrm{HCC}$ progression and metastasis $(5,10,11,16)$.

To the best of our knowledge, the present study was the first to perform expression profiling of miR-96 and miR-182 in non-metastatic HCC tissues. The two miRNAs were found to have similar expression levels in non-metastatic HCC tissues as compared to that in healthy liver tissues. However, when screened in the HuH-7 cell line, expression levels of the two miRNAs were significantly upregulated. Alongside these results, miR-96 and miR-182 were previously reported to be upregulated in metastatic tissues $(10,11)$.

Furthermore, there was a significant direct correlation between the expression of miR-96 and miR-182 in 22 HCC biopsy samples and in 10 healthy controls. This similar expression pattern may be explained by the fact that they belong to 

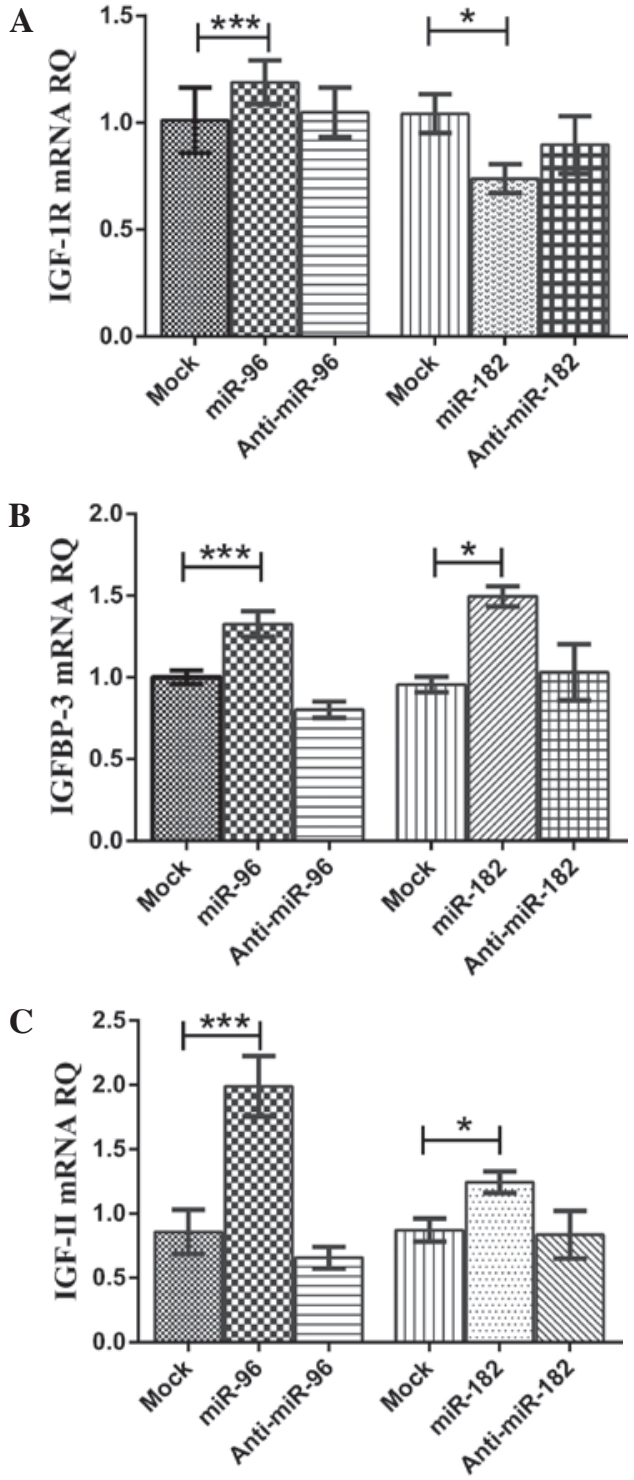

Figure 3. Impact of miR-96-5p and miR-182-5p on IGF-1R, IGFBP-3 and IGF-II transcript expression. (A) Transfection with mimics of miR-96-5p in HuH-7 cells resulted in a significant upregulation of IGF-1R mRNA levels compared to those in mock-transfected controls, while inhibitors of miR-96-5p caused a significant downregulation in the expression of IGF-1R transcripts compared to that in mimic-transfected cells. Mimicking of miR-182-5p in HuH-7 cells resulted in a significant downregulation of IGF-1R mRNA levels compared to those in mock-transfected controls, while inhibitors of miR-182-5p did not significantly change the expression of IGF-1R transcrip levels compared to those in mock-transfected cells. (B) Transfection of HuH-7 cells with miR-96-5p mimics resulted in a significant upregulation of IGFBP-3 mRNA levels compared to those in mock-transfected controls. However, inhibitors of miR-96-5p caused a significant downregulation in the expression of IGFBP-3 transcript levels compared with those in mock- and mimic-transfected cells. Transfection of HuH-7 cells with miR-182-5p mimics resulted in a significant upregulation of IGFBP-3 mRNA levels compared to those in mock-transfected controls. However, inhibitors of miR-182-5p did not cause any significant changes in the expression of IGFBP-3 transcript levels compared to those in mock-transfected cells. (C) Transfection of HuH-7 cells with miR-96-5p mimics resulted in a significant upregulation of IGF-II mRNA levels compared to those in mock-transfected controls. Of note, inhibitors of miR-96-5p caused a significant downregulation in the expression of IGF-II transcript levels compared to those in mock- and mimic-transfected cells miR-182-5p mimics caused a significant upregulation of IGF-II mRNA levels in HuH-7 cells compared to those in mock-transfected controls. However, inhibitors of miR-182-5p did not cause any significant change in the expression of IGF-II transcript levels compared to those in mock-transfected cells. The Mann-Whitney $\mathrm{U}$ test was performed. ${ }^{* * *} \mathrm{P}<0.001,{ }^{*} \mathrm{P}<0.05$. RQ, relative quantitation, IGFBP, insulin-like growth factor binding protein; IGF-1R, insulin-like growth factor-1 receptor; miR, microRNA. the same family (miR-96/182/183 family), which consorts with the findings of a previous study where a positive correlation was observed between miR-96, miR-182 and miR-183 expression in prostate cancer tissues (17).

By performing bioinformatics analyses, miR-96-5p and miR-182-5p were found to target the 5'-UTR and 3'-UTR of three crucial IGF axis genes in HCC, namely IGF-1R, IGFBP-3 and IGF-II transcripts. Therefore, the present study aimed at exploring the impact of miR-96 and miR-182 on IGF axis members to understand their role in $\mathrm{HCC}$ cancer progression.

The aberrant expression pattern of IGF axis members in human HCC tissues with overexpression of IGF-1R and IGF-II and downregulation of IGFBP-3 was reported to promote HCC progression and metastasis $(14,18,19)$.

Bioinformatics tools showed that miR-96-5p and miR-182-5p have the same potential binding sites in two regions of the 3'-UTR of IGF-1R mRNA. The two miRNAs were also predicted to target two different regions in the 5'-UTR of IGF-1R mRNA. In spite of the fact that miR-96 and miR-182 share an identical seed region (UUGGCA, nucleotides 2-7), the IGF-1R expression levels obtained upon transfection of HuH-7 cells with miR-96 and miR-182 mimics were not the same: In HuH-7 cells, IGF-1R mRNA was upregulated by miR-96 mimics and downregulated by miR-182 mimics compared to that in their respective mock-transfected cells. These findings are in agreement with those of another study, in which the glypican-3 (GPC3) 3'-UTR was predicted as a common target for the two miRs due to exhibiting one miR-96/182 binding site. miR-96 downregulated GPC3 expression by targeting its mRNA 3'-UTR; however, miR-182 failed to regulate it despite the seed similarity between the two miRNAs (20).

The second potential target, IGFBP-3 mRNA, was predicted to be targeted by the two miRs at the 5'- and 3'-UTRs. IGFBP-3 mRNA was found to be significantly upregulated upon ectopic expression of miR-96 in HuH-7 cells compared to that in mock-transfected cells, and the same results were observed upon transfection of $\mathrm{HuH}-7$ cells with miR-182 mimics. This overexpression of the downstream target was restored to normal levels upon antagonizing miR-96 and miR-182 using specific antagomirs in HuH-7 cells. The finding that the two miRNAs have the same effect on their target IGFBP-3 mRNA consorts with another study where the 3'-UTR of chloride intracellular channel 5, which contains a single miR-96/-182 binding site, was found to be directly targeted; however, in that case it was downregulated by the two miRs at the mRNA and protein level (21). The observed induction of IGFBP-3 mRNA by miR-96 and miR-182 may be due to 5'-UTR binding leading to stabilization of the mRNA, thus activating gene expression, which is considered a novel mechanism of action for miRs (22). This inducing impact was previously reported when miR-122 directly targeted two sites in the 5'-UTR of Hepatitis C virus RNA stimulating its translation and increasing its abundance (23-25).

Following the exploration of the effect of miR-96 and miR-182 on IGF-1R and IGFBP-3 mRNAs, the present study examined the effect of the two miR mimics on IGF-II mRNA, which was predicted to be targeted by both miRs at the 5'-UTR but not the 3'-UTR. Unexpectedly, it was found that IGF-II mRNA levels were significantly upregulated following transfection of HuH-7 cells with each of the miR mimics 
compared with those in their respective mock-transfected cells. The same enhancing effect on target mRNA by a miR was reported by a previous study on the receptor-interacting protein 140 (RIP140) mRNA, where miR-346 targeted the 5'-UTR of the RIP 140 transcript, leading to an upregulation of its protein levels (26).

This expression variability of IGF axis components upon miR-96 mimic-transfection raises a question about the simultaneous increase of IGF-1R and IGF-II, which are mitogenic, as well as IGFBP-3, which is anti-tumorigenic. This may be explained as a compensatory mechanism where the upregulation of IGF-1R and IGF-II may be neutralized by the upregulation of IGFBP-3, counteracting the tumorigenic effect of the mitogens and mediating their effect. This mediation by upregulated IGFBP-3 is realized through its binding to the overexpressed IGF-II, which inhibits the binding and consequent activation of IGF-1R and subsequent downstream signaling. By contrast, miR-182 has a dual role where it upregulates the mitogenic IGF-II and, as a compensatory mechanism, upregulates IGFBP-3 and downregulates IGF-1R to regulate the anti-mitogenic effect.

In conclusion, the present study showed for the first time, to the best of our knowledge, that miR-96 and miR-182 have similar expression levels in non-metastatic HCC tissues and in healthy controls, contradicting previous studies reporting their upregulation in metastatic tissues $(10,11)$. This highly implies that miR-96-5p and miR-182-5p are metastatic miRs in HCC. The present study also shows for the first time, to the best of our knowledge, the pleiotropic effect of miR-96-5p and miR-182-5p in targeting various components of the oncogenic IGF signaling pathway in HCC. Thus, these data provide the rationale for potential targeting of miR-96-5p and miR-182-5p in HCC therapy.

\section{Acknowledgements}

The authors acknowledge the patients and healthy volunteers for their valuable participation in this study. Furthermore, the authors would like to thank the Science and Technology Development Fund (STDF; grant no. 4242) for funding this work.

\section{References}

1. Baskerville S and Bartel DP: Microarray profiling of microRNAs reveals frequent coexpression with neighboring miRNAs and host genes. RNA 11: 241-247, 2005.

2. Guo H, Ingolia NT, Weissman JS and Bartel DP: Mammalian microRNAs predominantly act to decrease target mRNA levels. Nature 466: 835-840, 2010.

3. Selbach M, Schwanhausser B, Thierfelder N, Fang Z, Khanin R and Rajewsky N: Widespread changes in protein synthesis induced by microRNAs. Nature 455: 58-63, 2008.

4. Gottwein E and Cullen BR: Viral and cellular microRNAs as determinants of viral pathogenesis and immunity. Cell Host Microbe 3: 375-387, 2008.

5. Segura MF, Hanniford D, Menendez S, et al: Aberrant miR-182 expression promotes melanoma metastasis by repressing FOXO3 and microphthalmia-associated transcription factor. Proc Natl Acad Sci USA 106: 1814-1819, 2009.
6. Wang Y, Lee AT, Ma JZ, et al: Profiling microRNA expression in hepatocellular carcinoma reveals microRNA-224 up-regulation and apoptosis inhibitor-5 as a microRNA-224-specific target. J Biol Chem 283: 13205-13215, 2008.

7. Sato F, Hatano E, Kitamura K, et al: MicroRNA profile predicts recurrence after resection in patients with hepatocellular carcinoma within the milan criteria. PLoS One 6: e16435, 2011.

8. Ladeiro Y, Couchy G, Balabaud C, et al: MicroRNA profiling in hepatocellular tumors is associated with clinical features and oncogene/tumor suppressor gene mutations. Hepatology 47: 1955-1963, 2008.

9. Pineau P, Volinia S, McJunkin K, et al: miR-221 overexpression contributes to liver tumorigenesis. Proc Natl Acad Sci USA 107: 264-269, 2010.

10. Chen RX, Xia YH, Xue TC and Ye SL: Suppression of microRNA-96 expression inhibits the invasion of hepatocellular carcinoma cells. Mol Med Rep 5: 800-804, 2012.

11. Wang J, Li J, Shen J, Wang C, Yang L and Zhang X: MicroRNA-182 downregulates metastasis suppressor 1 and contributes to metastasis of hepatocellular carcinoma. BMC Cancer 12: 227, 2012.

12. Dong ZZ, Yao DF, Wu W, et al: Correlation between epigenetic alterations in the insulin growth factor-II gene and hepatocellular carcinoma. Chin J Hepatol 20: 593-597, 2012 (In Chinese).

13. Scharf JG, Schmidt-Sandte W, Pahernik SA, Ramadori G, Braulke $\mathrm{T}$ and Hartmann $\mathrm{H}$ : Characterization of the insulin-like growth factor axis in a human hepatoma cell line (PLC). Carcinogenesis 19: 2121-2128, 1998.

14. Gong Y, Cui L and Minuk GY: The expression of insulin-like growth factor binding proteins in human hepatocellular carcinoma. Mol Cell Biochem 207: 101-104, 2000.

15. Gray SG, Eriksson T, Ekstrom C, et al: Altered expression of members of the IGF-axis in hepatoblastomas. Br J Cancer 82: 1561-1567, 2000.

16. Xu D, He X, Chang Y, et al: Inhibition of miR-96 expression reduces cell proliferation and clonogenicity of HepG2 hepatoma cells. Oncol Rep 29: 653-661, 2013.

17. Yin Y, Li M, Li H, et al: Expressions of 6 microRNAs in prostate cancer. Natl J Androl 16: 599-605, 2010 (In Chinese).

18. Nussbaum T, Samarin J, Ehemann V, et al: Autocrine insulin-like growth factor-II stimulation of tumor cell migration is a progression step in human hepatocarcinogenesis. Hepatology 48: 146-156, 2008.

19. Aleem E, Nehrbass D, Klimek F, Mayer D and Bannasch P: Upregulation of the insulin receptor and type I insulin-like growth factor receptor are early events in hepatocarcinogenesis. Toxicol Pathol 39: 524-543, 2011.

20. Jalvy-Delvaille S, Maurel M, Majo V, et al: Molecular basis of differential target regulation by miR-96 and miR-182: the Glypican-3 as a model. Nucleic Acids Res 40: 1356-1365, 2012.

21. Gu C, Li X, Tan Q, Wang Z, Chen L and Liu Y: MiR-183 family regulates chloride intracellular channel 5 expression in inner ear hair cells. Toxicol In Vitro 27: 486-491, 2013.

22. Henke JI, Goergen D, Zheng J, et al: microRNA-122 stimulates translation of hepatitis C virus RNA. EMBO J 27: 3300-3310, 2008.

23. Villanueva RA, Jangra RK, Yi M, Pyles R, Bourne N and Lemon SM: miR-122 does not modulate the elongation phase of hepatitis C virus RNA synthesis in isolated replicase complexes. Antiviral Res 88: 119-123, 2010.

24. Norman KL and Sarnow P: Modulation of hepatitis $C$ virus RNA abundance and the isoprenoid biosynthesis pathway by microRNA miR-122 involves distinct mechanisms. J Virol 84: 666-670, 2010.

25. Jopling CL: Regulation of hepatitis C virus by microRNA-122. Biochem Soc Trans 36: 1220-1223, 2008.

26. Tsai NP, Lin YL and Wei LN: MicroRNA mir-346 targets the 5'-untranslated region of receptor-interacting protein 140 (RIP140) mRNA and up-regulates its protein expression. Biochem J 424: 411-418, 2009. 